\title{
Self-Regulated Learning in Students of The Faculty of Medicine and Surgery, Southeast Regional University (Oaxaca, Mexico)
}

\author{
García-Montalvo, I. A. ${ }^{1,2}$
}

\begin{abstract}
Introduction: Self-regulated learning is identified as an active, independent, autonomous, critical, adaptive, reflective and strategic process, to achieve comprehensive training, continuous selfimprovement, self-determination, autonomy and update itself permanently. The objective of this work is to analyze the self-regulation strategies of undergraduate students assigned to the Faculty of Medicine and Surgery of the Southeast Regional University for the 2019-2020 school year.
\end{abstract}

Methodology: This is a descriptive-cross-sectional study, the sample was obtained through a simple random sampling among students from first to fifth year, with substitution of exclusion elements, the instrument used was the adapted Torre questionnaire (2016) and validated for this study.

Results: Study subjects demonstrated adequate metacognitive awareness, control and verification, in addition to having active processing during classes, however, they present little effort in performing tasks, which leads us to establish recommendations for the educational practice that reinforces above all the teaching of these strategies in the university environment of health sciences.

Conclusion: The undergraduate students of the Faculty of Medicine and Surgery of the Southeast Regional University are clear about what self-regulated learning is mainly in students of more advanced years or who have already begun to contact the clinical area, unlike the students of the first years where behavioral strategies are still perceived.

Key words: Self-regulation, Health Sciences, Educational practice

\section{Introduction}

Self-regulated learning is a construct based on various theories that address aspects such as the reasons for self-regulation, the processes of self-awareness, the achievement of goals, the possible influence of the social and physical environment, and the acquisition of selfregulatory abilities.

${ }^{1}$ Faculty of Medicine and Surgery, Universidad Regional del Sureste, Oaxaca, Mexico

${ }^{2}$ Division of Postgraduate Studies and Research, Tecnológico Nacional de México/Instituto Tecnológico de Oaxaca, Oaxaca, México

Corresponding Author: Dr. Iván Antonio García-Montalvo, Faculty of Medicine and Surgery, Universidad Regional del Sureste, Oaxaca, Mexico. Libramiento Sur No.100 Esq, Hornos Ex-hacienda el Rosario San Sebastián Tutla, Oaxaca CP 68150

Email: ivan.garcia@catedraticos.urse.edu.mx

DOI: http://doi.org/10.4038/seajme.v14i2.279
It also incorporates effort management, planning, supervision and regulation of cognitive strategies and also motivation, behavior and the student's environment (Senko, 2011; Fernández, 2013).

Woolfolk (2010), defines self-regulated learning as the ability to learn to learn, learn to relearn, learn to unlearn, independently and autonomously, build individual paths to knowledge by giving meaning and meaning to what is learned, delving into the path of knowledge without taking the cognitive anchors of old reductionist prejudices and taking on new spaces to unleash the adventure of knowledge, dare to emerge in the midst of chaos, uncertainty, integrate processes that connote aspects of order and disorder (Woolfolk, 2010). Cognitive, metacognitive and resource regulation strategies are fundamental for the development of skills that allow the development of academic performance favorably. Among the cognitive strategies are 
the review, elaboration and organization strategies (Pintrich, 1990). In order to achieve academic success and optimal learning results, students need both skill and will, clearly reflecting the degree of interrelation between cognitive, metacognitive, self-regulatory, and emotionally motivational within school learning (Skaalvik, 1997; Suárez, 2016; Suárez, 2001), health science students can implement a series of motivational processes and learning strategies with which they build knowledge.

\section{Objective}

The objective of this study was to analyze the self-regulation strategies of undergraduate students assigned to the Faculty of Medicine and Surgery of the Southeast Regional University (Oaxaca, Mexico) corresponding to the 2019-2020 school year.

\section{Methods}

\section{Type of study}

It was a descriptive-cross-sectional study, applying a simple random sampling through Excel for students from first to fifth year.

\section{Data collection protocol}

For this work, a scale was used to determine the academic self-regulation adapted by Juan Carlos Torre in his doctoral thesis at the Universidad Pontificia Comillas Madrid (2006), designed for university students. This scale consists of twenty questions with five levels of response, which measured four factors:

- Factor I. Metacognitive awareness

- Factor II. Control and verification

- Factor III. Daily effort in completing tasks

- Factor IV. Active processing during classes

\section{Analysis of data}

The statistical analysis of the results will be carried out through descriptive statistics using the SPSS $\AA 24$ program and the generation of the database was carried out using Excel ${ }^{\circledR}$.

\section{Ethical aspects}

The study project, in the form of a protocol, was approved by the Research Committee of the Faculty of Medicine of the Southeast Regional University. The research units (students) signed the informed consent prior to entering the study, leaving them clear freedom in their decision to participate or not in the study. The information collected will be kept confidential and anonymous, it will be stored in a place with access only for the researchers in charge of the study.

\section{Results}

Sampling was performed from a universe of 900 students enrolled from first to fifth year of medicine, a confidence interval of 0.95 and 0.5 of probability of error was considered, resulting in a sample of 220 students. Of the sample, $31.6 \%$ were men and $68.4 \%$ were women, the average age of the study group was 21.8 years, with a minimum of 19 and a maximum of 27 years. Distributing between $32.2 \%$ of first year, $29.9 \%$ second year, $12.4 \%$ third year, $10.2 \%$ fourth year and $15.3 \%$ fifth year. For Factor I, it refers to the activation of pre-knowledge, and to be clear about what it pursues when learning a certain or certain content, that is why it is called metacognitive consciousness, the evaluated subjects presented $69.2 \%$ in favor of feeling identified with that activity while $13.18 \%$ are not clear on the item and $17.8 \%$ do not identify themselves or do not carry out this activity. For this first phase, preparation-planningactivation, it consists of determining the objectives, activating pre-knowledge and setting in motion processes of behavioral planning and the activation of perceptions related to both the task, the person and the context in which it is carried out. located, the study subjects are mostly clear about this objective when studying or preparing.

Factor II, includes monitoring, implies taking into account the metacognitive, emotional and behavioral awareness of the execution of the learning action; is to control effects, steps, or changes that occur in the different areas, also called control and verification, the evaluated subjects presented a $76.28 \%$ in favor of feeling identified with that activity while $6.64 \%$ are not clear on the item and on $17.09 \%$ does not identify or does not carry out this activity.

In factor III, control and regulation, means that by regulating yourself and not obtaining the expected results, you must adapt or change the cognitive and motivational strategies that you apply. In it, behavior and context are analyzed, the daily effort in carrying out the tasks, the evaluated subjects presented $66.45 \%$ in favor of feeling identified with this activity, while $15.15 \%$ are not clear on the subject and $18.5 \%$ do not identify themselves or do not carry out this activity.

Factor IV, reaction and reflection, involves the evaluation process, both of the teacher and of the student himself. And it is the reflection on the task, considers the emotional and behavioral reaction, looks for the active processing during the classes, the evaluated subjects presented $67.6 \%$ in favor of feeling 
identified with that activity while $15.46 \%$ are not clear about the item and $16.94 \%$ do not identify themselves or do not carry out this activity.

\section{Discussion}

Almost $70 \%$ of the undergraduate students of the Faculty of Medicine and Surgery of the Southeast Regional University can be considered as self-regulated students, taking into account that a self-regulated student is one who improves his academic performance by regulating his learning process, that is, when it takes into account: previous knowledge, zone of proximal development, context, motivation, feelings and emotions, it is frequently monitored when choosing learning strategies. Students must be able to take advantage of a variety of resources, in order to know how to process information, considering the various strategies they can use to achieve efficient and effective learning. Learning is not something that happens to him, it is something that can be caused by him, this implies that he must be monitored and regulated in the learning processes, aimed at achieving the proposed goals (Torre, 2007; Zimmerman, 2011). Torre (2007), claims that the characteristics of a regulated student are:

- Is aware that self-regulation is related to academic performance.

- Knows what their previous skills and knowledge are; This helps you choose the strategies that will allow you to learn efficiently and effectively.

- Know their emotions. Negatives know how to control them and thus achieve greater motivation.

- Knows how to choose self-regulatory strategies (cognitive, metacognitive, motivational and supportive).

- Monitors how he learns, constantly reviews his learning habits and strategies, changes if he deems it necessary.

- Manages to create favorable study and learning environments, taking into account the place, location, schedule, external and internal distractors.

- The self-regulatory strategies that you apply when learning, transfer them to your daily life.

The continuous improvement of the selfregulation process in undergraduate students depends not only on them but on the accompaniment-guide that the teacher can provide, they must be reflective, planners, critics, strategists, capable of discerning between the rigidity of traditional teaching and with the necessary intellectual maturity to direct the search for their own knowledge: raise awareness, dominate and use their personal abilities and adapt them to better strategies, in the process of building a new way of conceiving learning (Torre, 2007; Zimmerman, 2011).

\section{Conclusion}

In conclusion, we can say that undergraduate students of the Faculty of Medicine and Surgery of the Regional University of the Southeast are clear about what self-regulated learning is, mainly in students of more advanced years or who have already begun to contact the clinical area, to unlike the students of the first years where behavioral strategies are still perceived, although it is the responsibility of the students to improve their training process it also depends on the teacher since he plays an important role in the development and improvement of this activity, it is essential to carry out this type of detections in the first two years of school and determine the percentage of students that involve the self-regulation process with their academic performance, since this will open future studies that involve the present relationship between learning styles, selfregulated learning and academic performance, thus favoring the training of future doctors. The present relationship between Factor I and IV, can influence obtaining a better academic performance that is associated with the learning style developed by the students and with other factors. The first of them refers to the action that the teacher exercises in the learning process; the second is its ability to promote a comprehensive education in order to allow all students, with different self-regulatory styles, to achieve better learning results; the third refers to the characteristics of the faculty and the scaffolding that is offered through various mechanisms to stimulate autonomy in students, such as the implementation of a system of individual and group tutorials.

\section{References}

Fernández, E., Bernardo, A., Suárez, N., Cerezo, R., Núñez, J.C., Rosário, P. (2013). Prediction of use self-regulation strategies in higher education. Anal Psicología, 29, 86575.

Pintrich, P.R., de Groot, E.V. (1990) Motivational and self-regulated learning components of classroom academic performance. J Educ Psychol, 82, 33-40.

Senko, C., Hulleman, C.S., Harackiewicz, J.M. (2011) Achievement goal theory at the crossroads: Old controversies, current 
challenges, and new directions. Educ Psychol, 46, 26-47.

Skaalvik, E.M. (1997) Self-enhancing and selfdefeating ego orientation: Relations with task and avoidance orientation, achievement, self-perceptions and anxiety. J Educ Psychol, 89, 71-81.

Suárez, J.M., Fernández, A.P. (2016) El aprendizaje autorregulado: variables estratégicas, motivacionales, evaluación e intervención. UNED, Madrid, España.

Suárez, J.M., González, R., Valle, A. (2001) Multiple-goal pursuit and it's relation to cognitive, self-regulatory, and motivational strategies. Br J Educ Psychol, 71, 561-72.

Torre, J. (2007). Una triple alianza para un aprendizaje universitario de calidad. Madrid: Edit. Comillas, Madrid.

Woolfolk, A. (2010). Psicología educativa. (10 ed.). México: Pearson educación.

Zimmerman, B.J., Schunk, D. (2011) Selfregulated learning performance: An introduction and an overview. B.J. Zimmerman, D.H. Schunk (Eds.), Handbook of self-regulation of learning and performance, Routledge, New York, 1-15. 